\title{
La difficile question de l'universalité des droits de l'homme ${ }^{1}$
}

\author{
Geneviève Médevielle \\ Professeur d'éthique \\ Vice-Recteur à la Recherche
}

Plus de cinquante ans après la proclamation et la ratification de la Déclaration universelle des droits de l'homme par l'ONU, la violation de ces droits est finalement beaucoup plus universelle que leur reconnaissance. Les droits de l'homme ont pourtant permis de rendre plus exigeante la quête de justice et le devoir de la réaliser à l'égard de tous. Mais la question de leur fondement continue à se poser. Les uns, les conçoivent dans le cadre d'un rationalisme et humanisme laïc qui ne connaît pas d'autre fondement que l'autocompréhension d'une humanité autonome. Les autres pensent la véritable autorité des droits de l'homme à partir d'une transcendance. Car il ne suffit pas de présupposer l'égalité des hommes. Il faut introduire une instance absolue, appréhendée à travers un acte de foi ou par l'intermédiaire d'une conscience métaphysique. C'est devant cette instance extérieure à l'homme qu'on prend alors conscience d'une communauté de nature et de condition. Pour d'autres encore, l'énonciation des droits relèverait d'une appréciation individuelle et subjective de ce que chacun est en droit d'attendre des autres. Dans ce cas la prétendue rationalité de la morale des droits de l'homme se réduirait à un utilitarisme déguisé. Au bout du compte, dans nos sociétés occidentales pluralistes, la question des fondements des droits de l'homme reste l'objet d'un débat dont l'enjeu est la signification de la référence à la transcendance dans la pensée de l'universel en éthique.

Le soupçon et le doute sur la pérennité et l'universalité de ces droits se sont insinués dans les esprits. Même si ce corps de propositions a fait l'objet d'une large adhésion des Etats membres de l'ONU et s'il a été entériné par des législations positives, il n'est pas rare d'entendre reprocher aux droits de l'homme leur particularité régionale, historique et occidentale. Deux discours viennent disqualifier l'universalité des droits de l'homme. Le premier invalide des droits de l'homme au nom de l'histoire de domination et de colonisation de l'Occident. De fait, cette histoire a fait saisir l'abstraction du discours universel des droits de l'homme et le caractère fallacieux de l'émancipation des peuples promise par ces mêmes droits. La réalité de la discrimination qui demeure entre les membres de la communauté humaine vient ruiner l'appel à l'universel des droits de l'homme. Il y a

1 A Professora Doutora Genevieve Medevielle é Directora e Redactora Chefe da Revista Tranversalités - Instituto Caólico de Paris onde publicou uma primeira versão sobre a problemática dos Direitos do Homem em 2008. 
eu, il est vrai, beaucoup d'ethnocentrisme ${ }^{2}$ dans la manière occidentale de voir et d'imposer un type d'homme par la force et la violence. Mais, on ne peut pas se contenter d'enregistrer cette disqualification de l'éthique des droits de l'homme au nom du seul réalisme de la violence. Le détournement idéologique des droits de l'homme est un fait historique. Il ne saurait invalider l'instance critique que les droits de l'homme sont à l'égard de toute société. Le deuxième discours, tout aussi pernicieux pour l'universalité des droits, met l'accent sur l'idéologie bourgeoise qui les a façonnés ou qui perdure actuellement dans la manière qu'ont nos contemporains de se rapporter aux droits de l'homme. On part alors du constat que la référence aux droits de l'homme en Occident est très souvent au service de l'intérêt singulier et égoïste de l'individu. La «prétention » à l'universalité et à la permanence des droits de l'homme a donc besoin d'être défendue à l'occasion de la célébration du cinquantième anniversaire de la Déclaration Universelle des Droits de l'homme. L'intelligence de la foi elle-même doit être mobilisée dans la mesure où, du point de vue de la foi, ces droits incarnent l'exigence du respect de la dignité de tout être humain.

Dans cet esprit, nous allons nous attacher dans un premier temps à exposer comment on a pu en venir à douter de l'universalité des droits de l'homme. Sans faire l'historique de l'évolution, nous montrerons que malgré les faiblesses de certaines prétentions à l'universalité, la question de l'universalité reste posée. Comme l'a bien vu le théologien protestant Pierre Gisel, «une universalité humaine » est "à redéfinir, autant par-delà les différences culturelles et les divergences religieuses que par-delà une conception trop optimiste et trop occidentale de la raison et du progrès, de la laïcité ou de la sécularisation. » ${ }^{3}$. C'est pourquoi dans un deuxième temps, nous montrerons, face à cette situation, quelle contribution l'Eglise peut apporter non seulement à la compréhension de l'universel en morale, mais aussi à une compréhension de l'intégrité et de l'indivisibilité des droits de l'homme par l'affirmation qu'une dignité inaliénable est inhérente à tout être humain.

\section{La difficile reconnaissance des droits de l'homme}

Les bonnes intentions apologétiques ne suffisent pas à établir l'universalité des droits de l'homme. Certains confondent l'universalité et la pérennité en essayant de prouver que depuis toujours et partout ils ont existés. Il n'est pas rare de lire dans la

\footnotetext{
196.

2 Eric WEIL, « Du droit naturel » (1968), in Essais et Conférences, Paris, Vrin, 1991, t. 1, pp. 175-

3 Pierre GISEL, «Du concept de nature en théologie », in Eric FUCHS et Mark HUNYADI éd. Ethique et natures, Genève, Labor et Fides, 1992, p. 97.
} 
littérature contemporaine des attestations du règne des droits de l'homme dans le Code d'Hammourabi, dans la littérature gréco-latine, ou dans la littérature bouddhiste, dans le Coran ou encore dans la Bible. Très vite, on est proche soit d'un vague fondamentalisme soit d'un concordisme. Car comme l'a vivement souligné le juriste français Michel Villey, ces textes ne parlent pas des droits de l'homme même s'ils présentent une anthropologie cohérente avec la défense de l'éminente dignité de l'homme. «Les droits de l'homme sont nés dans l'Europe moderne. L'unité de la nature de l'homme et son éminence furent reconnues depuis les temps les plus reculés. Mais tout autre chose sont les droits de l'homme. ${ }^{4}$ Les idées de 1789 ont leur consistance propre. Et ce n'est pas par hasard si les Eglises chrétiennes et en particulier l'Eglise catholique, ont eu du mal à reconnaître les droits de l'homme marqués au coin d'un rationalisme subjectif. Ainsi, dans son radio-message de 1942, Pie XII se fait l'ardent défenseur « des droits fondamentaux de la personne $»^{5}$ sans pour autant assimiler la philosophie de 1789 incapable de rendre compte du lien intime existant entre les droits fondamentaux de la personne et les institutions naturelles et spontanées de sa vie sociale. Ce n'est donc pas en gommant les aspérités de l'histoire qu'on défendra le mieux l'universalité des droits de l'homme. Mais c'est peut-être davantage en examinant la généalogie historique de ces droits que nous pourrons préciser le rapport entre l'universalité et la pérennité des droits de l'homme et un légitime particularisme au sein même de ces droits.

Un examen un peu attentif de cette généalogie montre vite leur historicité et leur développement. Surgis en un lieu, en un temps et en une culture, les droits de l'homme ont bien la marque de l'Occident qui les a vu naître. Il est impossible de parler de pérennité des droits de l'homme au sens où ils auraient toujours été reconnus. Certes, les Anciens connaissaient la dignité de l'être humain. Mais l'idée des droits subjectifs et la conception d'un droit fondamentalement attaché à l'individu et déduit de sa nature sont nés dans la culture occidentale à une époque bien particulière : celle des Lumières. La tradition américaine et la Révolution française ont surtout formulé les grandes libertés individuelles et politiques, les « droits-attributs» de la personne individuelle, «les droits de... ». Avec ces droits, c'est toute une nouvelle conception de l'espace politique qui s'opère. Il est impossible désormais de se comprendre comme citoyens libres et égaux, pourtant divisés par des options morales, philosophiques et religieuses différentes, sans ce «noyau dur ${ }^{6}$ de valeurs et de règles de conduite valables pour tous. Il est frappant

${ }^{4}$ Michel VILLEY, Le droit et les droits de l'homme, Paris, PUF, 1983, p. 15-16.

5 On peut lire à ce sujet l'article de Juan-Miguel GARRIGUES, « La nature du droit fondement des droits de l'homme selon la doctrine catholique », Droits, Revue française de théorie juridique, PUF, ${ }^{\circ}$ 2, 1985, p. 45-59.

${ }^{6}$ L'expression est de la philosophe Anne FAGOT-LARGEAULT. En étudiant le pluralisme éthique de notre société occidentale dans le domaine procédural de la bioéthique, l'auteur note qu'il demeure 
de constater que la référence aux droits de l'homme reste la meilleure garantie ${ }^{7}$ pour un Etat de droit assurant paix, sécurité, liberté et égalité par et devant la loi, alors même que du point de vue éthique les références majoritaires deviennent celles du relativisme et du scepticisme. Les droits de l'homme sont, comme l'a démontré Eric Weil, « la norme du juste » de la démocratie occidentale.

A cette première génération de droits de l'individu face à l'Etat, la Déclaration universelle de 1948 ajoute les droits économiques et sociaux de la tradition socialiste du $\mathrm{XIX}^{\circ}$ siècle $^{8}$. Elle consacrait par ce geste l'évolution du contenu

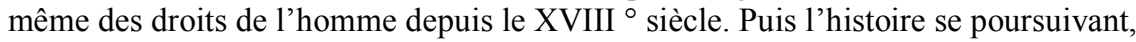
l'économie se mondialisant et mettant en danger l'avenir de tous et la survie des nations les plus pauvres, on a assisté dans les organisations internationales 9 au développement d'une troisième génération de droits, "les droits-solidarité » tels que le droit au développement, le droit à la paix internationale, le droit à la protection de l'environnement. La difficulté de l'extension du concept «droits de l'homme » a été soulignée par maints juristes. Au lieu de contribuer à leur garantie, l'extension du concept aboutissant à une prolifération des droits, affaiblit la notion même de droits de l'homme. Ils peuvent être alors identifiés à un souhait, une exigence ou une utopie. Dans le cadre de la troisième génération des droits de l'homme on cesse de voir précisément qui en est le titulaire, quel est leur objet propre, qui en est le débiteur, à qui ils sont opposables. C'est l'idée même de droit qui perd sa vigueur étant interprété à son tour comme un voeu non sanctionné ou un souhait inefficace. Un tel développement risque de mener à l'obscurcissement de ce qui fait leur unité. Michel Villey ${ }^{10}$ a souligné combien les déterminations de la morale des droits de l'homme sont comme souvent peu cohérentes, voire contradictoires et aporétiques. Du point de vue éthique, il y a aussi un risque à cette

« un noyau consensuel » ou «noyau dur » de l'éthique qui rejoint ce qu'on a coutume de désigner par l'expression «les droits de l'homme». Voir : Anne FAGOT-LARGEAULT, «La réflexion philosophique en bioéthique ", in Marie-Hélène PARIZEAU, Les fondements de la bioéthique, Bruxelles, De Boeck, 1992, p. 11-26.

7 Le philosophe politique Claude Lefort a parlé des droits de l'homme comme de la «charpente symbolique de la démocratie » dans la mesure où ils structurent l'espace politique en imposant au pouvoir politique de se référer aux citoyens et de rendre compte de ses décisions devant eux. Cf : Claude LEFORT, «Droits de l'homme et politique », L'invention démocratique. Les limites de la domination totalitaire, Paris, Fayard, 1981.

${ }^{8}$ La reconnaissance de ces droits impose un glissement sémantique du droit à la liberté tel que la déclaration de 1789 pouvait le stipuler. Désormais, ce droit se fonde dans l'idée même de fraternité humaine remise en lumière par l'art. 1 de la Déclaration universelle : "Tous les êtres humains naissent libres et égaux en dignité et en droits. Ils sont doués de raison et de conscience et doivent agir les uns envers les autres dans un esprit de fraternité. »

9 La préoccupation pour les droits de l'homme se poursuit au sein de plusieurs institutions, notamment dans le contexte du Conseil de l'Europe et de la Conférence sur la Sécurité et la Coopération en Europe.

10 Michel VILLEY, Op. cit, 1983. 
prolifération des droits : celui de la dérive d'une référence utilitariste aux droits de l'homme. Le point de vue d'une liberté et des attentes légitimes de l'individu l'emporte alors sur la rationalité des obligations et devoirs valables pour tous.

Le développement historique du contenu des droits que nous venons d'examiner va de pair avec l'évolution de la référence à ce qui les fonde. Ainsi « la Déclaration Universelle des Droits de l'Homme » de 1948 a été solennellement proclamée et ratifiée sans faire référence à Dieu ou au droit naturel ${ }^{11}$ à la différence de la première génération des Déclarations du XVIII ${ }^{\circ}$ siècle. Cette disparition du fondement n'est pas sans conséquence comme le relevait Léo Strauss en 1953 : « rejeter le droit naturel revient à dire que tout droit est positif, autrement dit que le droit est déterminé exclusivement par les législateurs et les tribunaux des différents pays. Or, il est évident qu'il est parfaitement sensé et parfois même nécessaire de parler de lois et de décisions injustes. En posant de tels jugements, nous impliquons qu'il y a un étalon du juste et de l'injuste qui est indépendant du droit positif et qui lui est supérieur : un étalon grâce auquel nous sommes capables de juger du droit positif. » 12

Rappelons que le respect accordé aux droits de l'homme déclarés par les révolutionnaires américains et français était solidaire d'une décision philosophique : si ces droits ne sont pas l'expression d'une norme absolue de justice, les droits exposés sont arbitraires et n'ont aucune valeur obligatoire pour l'autorité politique en place. La question décisive était donc celle de l'origine transcendante de ces droits, au sens de «ce n'est pas nous qui en décidons ». D'où l'importance non seulement de déclarer ces droits comme «naturels, inaliénables et sacrés», mais aussi de les reconnaître « en présence et sous les auspices de l'Etre suprême ».

Si tel est bien le ressort philosophique commun des premières déclarations, il y a néanmoins une différence entre la déclaration française et celle de Virginie. Le droit naturel «reconnu» dans la déclaration française de 1789 ne s'identifie pas exactement à celui de l'héritage américain. Lorsque John Locke dans son livre Le deuxième traité du gouvernement civil parle du droit naturel, c'est toujours sur un horizon théologique où Dieu est conçu comme le Créateur de la nature. «La loi de nature est une règle éternelle pour tous les hommes, législateurs ou non. Les normes édictées par les législateurs doivent se conformer à la loi de nature, c'est-à-dire à la

11 Le droit naturel au sens de l'idée d'un droit qui respecte une règle de nature et qui a ainsi la prétention à l'universalité de la norme de justice proclamée. Cf : Blandine BARRET-KRIEGEL, Les droits de l'homme et le droit naturel, Paris, PUF (coll. Quadrige), 1989, p. 41.

12 Léo STRAUSS, Droit naturel et histoire, (1953) trad. française de Nathan et Dampierre, Paris, Flammarion, (coll. Champs 158), 1969, p. 14. Il ne peut être question ici de refaire tout l'historique de la tombée en désuétude du droit naturel dans la tradition des droits de l'homme. Notons seulement que la norme de justice de ces droits a changé en un siècle et que cette transformation est dépendante du mode de « reconnaissance » de ces droits. 
volonté de Dieu, dont elle est une déclaration. »13 C'est dans cette ligne que s'inscrit la déclaration américaine. En revanche, en France, l'horizon théologique qui fonde la compréhension du droit naturel est déjà brouillé ${ }^{14}$. Lorsque la déclaration française fait intervenir le droit naturel, il ne s'agit pas tant d'en venir à poser «c'est Dieu qui fonde ces droits" que de faire exister, par engagement responsable, un état social et politique. Son origine est l'obéissance à des principes éthiques qui indiquent ce qui doit être désormais. Déjà dans la France de 1789, les autorités politiques ne peuvent plus, comme dans les Etats-Unis protestants, brandir la révélation biblique comme parole originaire, évidente et contraignante pour tous. Même si l'ambiguïté demeure et que l'Etre suprême peut être compris en référence au Dieu biblique, la référence explicite est déjà brisée. On ne se situe que « sous les auspices » de l'Etre suprême, témoin neutre et distant, et non pas dans l'obéissance à l'instance fondatrice du droit naturel, tel que le Dieu créateur et donateur de la loi fonctionne dans la théorie de Locke et dans le texte américain de 1776. Le sujet du droit, ce n'est pas Dieu mais la volonté des représentants du peuple au sens de la volonté générale chez Rousseau.

En proclamant les «Droits de l'Homme » comme obligations inconditionnelles pour tous, les révolutionnaires de 89 affirmaient que les rapports moraux entre les hommes n'ont à être déterminés qu'en fonction de ce qui leur est commun et qui les rend tous semblables. C'est dire que les "droits naturels » ne dépendent que de l'acte déclaratif qui les enregistre. L'acte juridique de la Déclaration de 1789 a donc un curieux statut : il se réclame d'une norme de justice du droit naturel, la reconnaît, la reformule sans pour autant la lier explicitement à une instance fondatrice. Le rapport théologico-politique de l'homme à Dieu est évacué. Dès lors, comme le fait remarquer Blandine Barret-Kriegel : « Les droits naturels ne dépendent pas de la loi naturelle mais de l'acte déclaratif qui les enregistre. Il n'y a plus de loi naturelle. La Déclaration de 1789 est une déclaration conforme au droit naturel mais non soumise à la loi naturelle. " 15 Finalement, la référence au droit naturel déliée de la loi naturelle reste dans l'indécision et ne joue plus pour le lecteur de la Déclaration qu'à l'état de trace indirecte liée à la reconnaissance des rédacteurs.

C'est dire la richesse et la fragilité de la référence au droit naturel dans la Déclaration de 1789. D'un côté, cette référence atteste la juridicité universelle des droits conformes à la nature de l'homme. Droits et devoirs ne sont plus à penser relativement à ce que les hommes d'une société particulière appréhendent comme

13 John LOCKE, The Second Treatise of Civil Government, New York, Hafner, 1947, p. 190.

14 La contribution de Jacques Maritain au discernement de la polysémie du concept «droit naturel » au XVIII ${ }^{\circ}$ siècle peut être utilement consultée. Cf : Jacques MARITAIN, «Théories de la loi naturelle » ( $4^{\circ}$ leçon donnée en 1950 dans le cadre d'un cours d'été pour l'Eau Vive), La loi naturelle ou loi non écrite, texte établi par G. Brazzola, Fribourg, Editions universitaires de Fribourg (coll. Prémices), 1986, p. 79-103.

15 Blandine BARRET-KRIEGEL, Op. cit., 1989, p. 24. 
juste, mais sont à déduire d'un homme doué de raison, universel et abstrait. Indépendamment de l'affirmation de droits particuliers relatifs à un pays, une culture ou une époque, les philosophes des Lumières avaient souligné la suprématie des droits et devoirs relatifs à tout individu en tant que dépositaire des caractères génériques de l'humanité. D'un autre côté la référence au droit naturel n'existe qu'en vertu de l'acte de déclaration. Dès lors, même si l'instance déclarative ne se substitue pas à l'instance fondatrice et continue de s'en distinguer, un espace est libéré pour penser plus faiblement la fondation des droits de l'homme que par la référence à une même condition de dépendance à l'égard d'une transcendance ${ }^{16}$. C'est ce dont témoigne, un siècle et demi plus tard, la Déclaration de l'ONU.

La Déclaration de 1948 est fondée sur la "considération» historique des « horreurs » de la barbarie nazie et propose de s'appuyer sur la foi dans « la dignité et la valeur de la personne humaine ». Le personnalisme devient le substitut du droit naturel du XVIII ${ }^{\circ}$ siècle ${ }^{17}$. Mais la dignité humaine a-t-elle ce caractère de principe inviolable et sacré qui était attribué aux droits naturels de 1789 ? L'obligation éthique de respecter la personne est à la mesure de ce dont il est fait mémoire : l'expérience historique d'un régime de mort et de barbarie qui correspond au déni de la dignité de tout être humain. C'est par la médiation de l'expérience négative et de sa mémoire que l'humanité entend fonder les droits de l'homme sur la « foi » 18 en la dignité de la personne humaine. S'ouvre là un moyen d'attester l'humain dans son universalité sans se référer à un fondement transcendant. La reconnaissance des droits de l'homme se fait indirectement par l'expérience même de leur déni au coeur de la barbarie, du génocide et de la terreur. Il s'agit là d'un jugement moral de la sagesse pratique qui, sur fond d'horreur historique, discerne les chemins qui conduisent à la vie et ceux qui conduisent à la mort.

$\mathrm{Au}$ départ de cette reconnaissance indirecte, il y a une expérience concrète, négative $^{19}$, de souffrance, d'injustice et de perdition humaine qui provoque

16 Jacques Maritain a été un des premiers philosophes catholiques à réfléchir cette situation paradoxale, quand il soulignait que la reconnaissance et la formulation des droits de l'homme pouvaient faire l'objet d'une entente pratique alors que l'accord rationnel sur leur fondement pourtant nécessaire devenait impossible, voire insurmontable.

17 Avec l'abandon du droit naturel, la Déclaration de l'ONU ne peut pas être aussi claire en ce qui concerne la différence de niveaux entre les droits fondamentaux et les droits civils. Alors qu'au XVIII ${ }^{\circ}$ siècle les premiers étaient référés à la volonté du Créateur, et les droits civils étaient référés à la volonté générale du peuple souverain.

18 L'expression appartient en propre au $4^{\circ}$ considérant de la Déclaration universelle des droits de l'homme de 1948 : « les peuples des Nations unies ont, dans la Charte, proclamé à nouveau leur foi dans les droits fondamentaux de l'homme, dans la dignité et la valeur des hommes et des femmes ».

19 Deux théologiens, E. SCHILLEBEECKX et Dietmar MIETH, ont mis en valeur cette expérience de négativité où se jouent la découverte d'une valeur et l'implication éthique à la faire advenir. Le «contraste» in E. SCHILLEBEECKX, «Portée théologique des déclarations du Magistère en matière 
l'attention des survivants et mobilise alors leur mémoire des aspirations les plus élevées de l'humanité ${ }^{20}$. Dans le cas présent, c'est la mémoire de l'inscription historique de ces aspirations en Occident qui aide à diagnostiquer les manquements et à attester les possibilités d'accomplissement d'une vie humaine. Car si dans la mémoire de l'expérience de négativité et de souffrance, il y a perception et connaissance que certaines possibilités de vie ne sont pas remplies, c'est qu'un processus d'interprétation est à l'oeuvre par rapport soit à d'autres situations historiques où la vie est rendue possible, soit plus fondamentalement à une promesse d'existence ${ }^{21}$.

Mais suffit-il pour fonder ces droits de se référer à une généalogie historique des protestations en faveur de la liberté et de la dignité humaine ? Aucun sage, comme l'a souligné le théologien protestant Eric Fuchs ${ }^{22}$, ne peut fonder sur de simples faits de vie historiques que la vérité et le bonheur, la justice et la liberté, la vie et l'avenir soient possibles. Trop d'échecs et d'épreuves viennent faire constater le contraire. Pourtant, c'est ce que l'on peut «croire» et fonder en vertu d'une promesse d'existence. Car d'où peut venir cette confiance, sinon de cette promesse existentielle fondamentale signifiée dès la naissance par l'entrée de tout être humain dans une communauté de parole qui l'invite à vivre et à croire qu'un avenir lui est ouvert? Autrement dit, si le discernement et l'interprétation d'une nouvelle norme d'existence peuvent se jouer au coeur de la négativité, c'est bien en vertu de cette promesse qui se transmet à travers le simple fait d'être mis au monde. Parce qu'elle est ouverture à un avenir authentique, la promesse d'existence comporte en-elle même, une force d'obligation : celle de ne pas faire obstacle à ce que peut et doit devenir cette promesse de vie et d'humanisation pour chacun. C'est dire que le respect des personnes promu par les droits de l'homme correspond au respect de la destinée de cette promesse d'existence pour chaque être humain. Ce respect de la destinée de la promesse suppose donc la reconnaissance de la place des autres et la nécessité de respecter en eux ces exigences auxquelles nous ne voudrions pas renoncer pour nous-mêmes.

Le drame historique de la transmission de la promesse c'est qu'en certaines cultures le concept d' « autres » se restreint à certains groupes. Il devient impossible de témoigner que tous peuvent espérer en un avenir. C'est ce que souligne le philosophe Eric Weil dans son article sur le droit naturel : «Le groupe traditionnel

sociale et politique », Concilium, n 36,1968 , p. 27-44 ; D. MIETH, «Plaidoyer pour un modèle éthique », Concilium, n 120,1976, p. 34-35.

$20 \mathrm{Cf}: 2^{\circ}$ considérant de la Déclaration universelle des droits de l'homme de 1948.

21 L'évocation de la genèse historique de la Déclaration des droits de l'homme de 1789 est à ce titre exemplaire. Sur fond des Cahiers de doléances, la déclaration française se rattache bien à une expérience historique négative de déni de droits pourtant déjà positivement expérimentés en terre d'Amérique.

22 Eric FUCHS et Pierre-André STUCKI, Au nom de l'Autre. Essai sur le fondement des droits de l'homme, Genève, Labor et Fides, 1985, en particulier p. 101-124. 
ne connaît pas d'obligation envers ceux qui lui sont extérieurs, les ennemis naturels, les étrangers, les sous-hommes, ces êtres qu'on a le droit, souvent l'obligation, de soumettre, de réduire en esclavage, d'offrir en sacrifice à ses divinités : c'est dans l'histoire que l'idée "ethnocentrique" de l'universalité est devenue elle-même universelle en ce sens qu'elle n'est plus l'apanage d'un groupe. » ${ }^{23}$ En effet, si la particularité d'une tradition, au lieu de s'ouvrir à la transmission de la promesse d'humanisation valable pour tous, se pose comme l'émetteur de celle-ci, l'avenir des personnes se ferme sur une probabilité ou une habitude incontestée, au mépris de la liberté, de la dignité et de l'égalité fondamentale de tous.

Finalement, lorsque celui qui fait mémoire des horreurs de la barbarie occidentale, se pense en «solidarité-responsabilité » avec d'autres humains - tout particulièrement en solidarité avec les victimes - un universel est attesté. Il s'agit d'un universel concret qu'on ne peut pas identifier à un invariant tel que l'universalisme moral traditionnel le postulait par la découverte-présence de traits communs à toute l'humanité. Il s'agit ici d'un universel qui permet de fonder radicalement la possibilité d'une critique sociale et culturelle au nom d'une réduction de l'humain que fait courir toute particularité incapable de transmettre à chacun(e) la possibilité d'une vie vraiment humaine. Autrement dit, ce n'est pas la particularité, la contingence historique et culturelle en tant que telle qui est contestée, mais c'est sa capacité à donner expression à la réalisation de l'humain dans la personne humaine. A l'universalité abstraite du rationnel, se substitue un universalisme concret déterminé par « la sphère cachée des intentions véritables de la vie $»^{24}$, pour parler comme Vaclav Havel. A un certain degré d'injustice, de souffrance et de malheur, l'émergence de la vérité de l'humain réclamant les conditions d'une survie bouscule l'ordre des valeurs reçues. Mais cette mise en cause de l'emprise communautaire particulière pour la définition de l'humain n'est pas sans ambiguïté. La critique de l'emprise communautaire demande d'être normée. La Déclaration de 1948 pose la norme du respect absolu « de la dignité inhérente à tous les membres de la famille humaine ». Cette postulation éthique ne suppose pourtant pas une fondation théorique unanime. Elle peut être affirmée avec force à partir de l'expérience de la négativité, sa justification n'en demeure pas moins plurielle et cette pluralité s'avère dans la Déclaration universelle insurmontable.

A cette faiblesse de la fondation des droits de l'homme à partir de la négativité, il faut ajouter l'ambiguïté du modèle de réciprocité qui fonctionne dans l'identification à la victime. Le sujet reconnaît le mal qui a été fait à des êtres humains dans l'histoire et s'engage à ne pas le réitérer à partir du ressort de son identification aux victimes. Le cas n'est pas académique ou abstrait. Certains

\footnotetext{
23 Eric WEIL, « Du droit naturel » (1968), Essais et Conférences, Paris, Vrin, 1971, tome 1, p. 191192.

24 Vaclav HAVEL, Essais politiques, Paris, Calmann-Levy, 1989, p. 89-91.
} 
tenants de l'éthique des droits de l'homme ou de l'euthanasie comme droits au nonmal (d'être maltraité, offensé, meurtri...) ${ }^{25}$, refusant l'idée d'une identité naturelle ou spirituelle de l'homme, ne peuvent trouver la norme de leur action que par projection-identification à la victime. Mais cette identification ne va pas de pair avec le respect dû aux victimes commandé par la précompréhension de la dignité de cette personne. Or, c'est une chose de faire mémoire des victimes, c'est autre chose que de définir l'homme selon ce statut. La tradition chrétienne nous rend attentifs à ce maniement ambigu de "l'homme comme victime». Les récits de la vie des camps, tel celui du Père Jacques Sommet s.j. à Dachau, nous présentent des hommes, qui ont été tout autre chose que des victimes promises à l'abattoir, de simples «êtres-pour-la-mort ${ }^{26}$. Les théologiens qui ont parlé du souvenir des victimes l'ont fait au nom de leur foi et de la «réserve eschatologique ». JeanBaptiste Metz en pointant vers le sujet humain comme contemporain de la fin de l'histoire ne voit pas la victime comme un pur " matériau » de cette histoire mais comme un sujet devant la face de Dieu. Il est tout autre chose qu'un simple mortel. La victime, l'exclu est le signe que le programme politique, économique ou juridique est préféré à la dignité de l'être humain. Le concept de dignité humaine exigerait de ne pas être évacué d'une réflexion sur la mémoire des victimes, si l'on veut faire droit à la reconnaissance absolue et intrinsèquement universelle du respect de tout homme.

Un des points les plus clairs à retenir de cette histoire contrastée, c'est que la reconnaissance des droits de l'homme se présente sous le mode déclaratif d' " un dit-de-justice ». Sous la modalité de la description, de la promesse et de l'appel, qui orienteront une éthique des droits de l'homme déontologique (1789) ou téléologique (1948) le dit-de-justice atteste d'une précédence de la norme de justice par rapport au droit positif et invite à agir en adéquation avec celle-ci. La reconnaissance de la précédence d'une norme de justice non-arbitraire fonde la «prétention» d'universalité de ces corpus normatifs historiques. Elle garantit la légitimité du lien social. Elle indique que le pouvoir même des lois n'est pas à la libre disposition de ceux qui les font et elle exige sa réalisation à travers le respect et la promotion de la dignité de tout être humain. Qu'il s'agisse du droit naturel ou de la promesse de vie pour chacun, l'exigence de la validité du juste et du nonarbitraire est indispensable pour fonder la pertinence des droits de l'homme. Car le

25 Voir l'éthique de Peter SINGER, Questions d'éthique pratique, Paris, Bayard éditions, 1997.

26 «Qu'y avait-il sous l'éphémère et terrible existence? Telle était l'unique question. Par sa réponse, la liberté de chacun posait l'acte qui l'engageait tout entière, et d'une façon particulière à chacun, dans la confiance ou le désespoir, la foi ou la sérénité, pour le temps qui lui restait à vivre. Par là, un sens fut donné aux décisions comme aux refus, qui les colore indépendamment de leurs effets apparents.[...] Quoi d'autre nous importe, après tout, que de mieux connaître en l'homme ce qui le rend assez maître de son destin pour dominer ainsi et la mort et la vie : cela seul intéresse, à travers les contemporains de Dachau, l'homme de tous les temps. " Jacques SOMMET, "Liberté à Dachau ", Christus, $\mathrm{n}^{\circ}$ 94, avril 1977, p. 242. 
risque est constant d'enfermer cette exigence dans les formes qu'elle peut prendre avec le temps.

Mais les équivoques pesant sur l'utilisation du droit naturel délié de la loi naturelle en 1789 et sur la mémoire de l'irrespect des victimes ne peuvent pas être évacuées. Le caractère toujours précaire des droits de l'homme, signalé précédemment, renvoie à la diversité des conceptions de la justice qui sont en jeu. Elles peuvent se contredire, se soutenir ou s'articuler selon le jeu des interprétations possibles des textes. Le modèle de justice qui semble l'emporter est celui de la « conception formaliste » telle que l'a formulée Pierre Bühler : "Si le scandale de l'injustice est pris au sérieux, la justice ne se trouve plus simplement être un résultat de l'ordre des faits constatables. Plutôt que de se vérifier de facto, elle s'articule comme lutte contre l'injustice régnant de facto. Cette conception ne se contente pas de décrire l'état donné comme l'état juste ; elle décrète ce qui doit être considéré comme juste et réfléchit par là au comment de l'engagement dans la réalité, plutôt qu'au quoi de la réalité elle-même. Elle énonce un certain nombre de principes élémentaires, de caractère formel et fixés à priori. » ${ }^{27}$ S'il y a lieu de penser que ce modèle est solidaire en 1789 d'un modèle « originaire » 28 de justice, celui du droit naturel, cette solidarité ne va pas de soi pour 1948. En revanche, ce que nos deux déclarations supposent, c'est, comme l'a souligné $P$. Bühler, que «la justice se présente comme une parole adressée à l'homme, lui annonçant qu'il peut recevoir une réponse à sa quête et l'appelant à saisir cette réponse, à se l'approprier et à en vivre. ${ }^{29}$. Une conception «dialogique» de la justice est à l'oeuvre dans ces déclarations. Mais l'instance du dialogue n'est pas toujours reconnue par tous. C'est là qu'il faut prendre position de manière théologique sur l'apport original de la foi chrétienne au modèle dialogique de justice des droits de l'homme. En effet, comme l'affirme Jean-François Collange «la geste des Droits de l'homme pourrait bien ne pas renvoyer à moins qu'à la genèse fondamentale de toute humanité, comme à la prévenance de l'Autre qui la suscite et à sa Parole qui la génère. »30

27 Pierre BÜHLER, « Introduction » à Justice en Dialogue, Genève, Labor et Fides, 1982, p. 16.

28 Le modèle est originaire dans le sens où le dit-de-justice est reconnu comme antérieur et précédant toute déclaration positive. En ce sens d'une précédence de principe, ce modèle est celui du droit naturel.

29 Pierre BÜHLER, art. cit., p. 17.

30 Jean-François COLLANGE, Théologie des droits de l'homme, Paris, Cerf, (coll. Recherches morales), 1989, p. 13. 


\section{La marque de l'universel et la dignité de l'homme}

Personne ne conteste la défense concrète des droits de l'homme par l'Eglise catholique au nom de sa foi ${ }^{31}$. L'exigence de justice n'est réservée à aucun groupe humain privilégié. Mais ce qui fait question, c'est la possibilité d'une fondation théologique de ces droits issus d'une tradition laïque ${ }^{32}$. Le débat n'est pas nouveau. Déjà dans La Revue des Deux Mondes, de 1891, Anatole Leroy-Beaulieu affirmait contre un courant laïc et socialiste : "L'idée de droits, inhérents à la personne de l'homme, est en réalité une idée chrétienne. $»^{33}$ Aujourd'hui encore, il n'est pas rare de lire que c'est notre tradition chrétienne qui peut donner sa force à l'affirmation des droits universels de $1^{1}$ 'homme ${ }^{34}$. Face à cet essai pour fonder théologiquement les droits de l'homme, certains crient à la récupération facile ${ }^{35}$, voire à «l'opportunisme clérical ${ }^{36}$. C'est dire qu'en entrant sur le terrain de la contribution chrétienne à l'universalité des droits de l'homme, nous entrons sur un terrain conflictuel ${ }^{37}$. Si nous étions tentés par une corrélation irénique entre la raison et la foi, nous pourrions nous satisfaire du nouveau paradigme de l'universalité des droits de l'homme de 1948. Mais à cause de la fragilité du fondement de la dignité humaine par l'effet de la seule mémoire de la négativité, il faut pouvoir travailler à fonder vigoureusement l'impératif éthique des droits de l'homme. Or la tradition théologique catholique n'est pas démunie d'arguments tant

31 L'attitude d'Amnesty International est ici exemplaire lorsque cet organisme accueille en son sein croyants et non-croyants en se défendant d'imposer à ses membres une conception anthropologique déterminée.

32 Cf : Jean-Louis SCHLEGEL, "Table ronde sur les fondements des droits de l'homme », Actes du Colloque Justice et Paix et Département de la recherche de l'Institut catholique de Paris, Les catholiques et les droits de l'homme. Débats et enjeux, Paris, Commission française Justice et Paix, 1992, p. 12-15.

33 Anatole LEROY-BEAULIEU « La papauté, le socialisme et la démocratie » (15 décembre 1891 et 15 janvier 1892), La Revue des Deux Mondes, mai 1991, p 63.

34 Cf : Eric FUCHS et Pierre-André STUCKI, Op. cit., 1985, p. 125 : «Les droits de l'homme ne peuvent vraiment être reconnus dans leur portée ultime que si on les comprend comme l'expression même de la loi de Dieu. »

35 Un bon exemple de cette tentation récupératrice nous est donné par le texte « Chrétiens et droits de l'homme » diffusé par les Commissions tiers-monde des Eglises protestante et catholique de Genève en novembre 1978, où chaque article de la Déclaration de 1948 est commenté par des versets bibliques ainsi que par des déclarations officielles des Eglises.

36 Juan-Miguel GARRIGUES, «La nature du droit fondement des droits de l'homme selon la doctrine catholique », Droits, Revue française de théorie juridique, PUF, n 2, 1985, p. 45.

37 Cf. l'essai très bien documenté de G. THILS, Droits de l'homme et perspectives chrétiennes, Louvain-la-Neuve, 1981, (Cahiers de la Revue théologique de Louvain, ${ }^{\circ}{ }^{2}$ ). 
rationnels qu'évangéliques pour affirmer l'éminente dignité de tout homme ${ }^{38}$. C'est le paradoxe du christianisme de prétendre asseoir la valeur universelle de tous les êtres humains sur un fondement particulier : celui de la Révélation qui en JésusChrist «permet à quiconque de recevoir le mystère de sa vie » ${ }^{39} \mathrm{La}$ fondation théologique des droits de l'homme n'est pas alors entreprise en vue de « récupérer » théologiquement les textes des Déclarations, ni de leur fournir après-coup un surplus de sens, ni encore d'en faire une transcription de l'Evangile.

Aux yeux des historiens, il est clair qu'il aura fallu près de deux cents ans à l'Eglise catholique pour s'engager dans la défense pratique et théorique des droits de l'homme. Mais depuis l'Encyclique Pacem in terris et le pontificat de Jean-Paul II, une attention toute particulière a été accordée à la sauvegarde et à la promotion de la dignité de la personne et de ses droits. C'est avec le pontificat de Jean XXIII et spécialement l'encyclique Pacem in terris 40 que l'intégration des droits de l'homme à l'enseignement social de l'Eglise se réalise presque comme allant de soi en continuité avec la doctrine de la loi et du droit naturels. Ainsi Pacem in terris atteste - au sens où l'on se porte témoin devant Dieu et devant les hommes - d'une universalité fondée sur la notion biblique de Création. Pour délivrer ces droits de l'arbitraire et du relativisme, l'encyclique reprend le geste de la Déclaration américaine qui nomme l'instance fondatrice des droits : à savoir le Créateur. Mais, tout en se réclamant de la Révélation, cette première charte ecclésiale des droits de l'homme, entend pouvoir se faire comprendre de tous grâce au concept de «nature humaine ». « Nature », « droits de nature », « nature humaine », « ordre juridique voulu par Dieu» et «loi naturelle» forment alors la constellation de mots indispensables pour rendre compte de la destinée de la personne humaine et de l'universalité des droits de l'homme.

Le discernement des droits et des devoirs de l'homme et leur nécessaire articulation qu'opère Pacem in terris, se fonde alors sur une conception de « l'ordre de l'univers » tel que la Révélation le donne à découvrir et à contempler ( $\left.\mathrm{PT} \mathrm{n}^{\circ} 2\right)$. Ordre de la Création dont la grandeur de l'homme est de pouvoir le saisir comme l'expression du projet de Dieu inscrit au plus intime de la nature (PT $\left.\mathrm{n}^{\circ} 5-6\right)$. Ainsi,

38 La dernière encyclique de Jean-Paul II, Fides et ratio redit avec force et sobriété qu'au point de départ de toute réflexion que l'Eglise entreprend «il y a la conscience d'être dépositaire d'un message qui a son origine en Dieu même. La connaissance qu'elle propose à l'homme ne lui vient pas de sa propre spéculation, fût-ce la plus élevée, mais du fait d'avoir accueilli la parole de Dieu dans la foi » JEAN-PAUL II, Fides et ratio, Rome, 14 septembre 1998, §7. Mais la morale en régime chrétien ne peut se passer d'une autre source, la source rationnelle, source antérieure à la révélation et parallèle à elle qui permet à tout homme de découvrir par eux-mêmes le contenu de la loi morale ou autrement dit ce qui est humanisant en «lisant le témoignage de l'être, de la vérité, du bien et de la beauté que les créatures possèdent en elles-mêmes. » JEAN-PAUL II, Audience du 27 mars 1985, Documentation catholique, $\mathrm{n}^{\circ}$ 1895,5 mai 1985 , p. 480.

39 JEAN-PAUL II, Fides et ratio, $§ 15$.

40 JEAN XXIII, Pacem in terris, Rome, 9 avril 1963. 
« tout droit essentiel de l'homme emprunte sa force impérative à la loi naturelle, qui le donne et qui impose l'obligation correspondante » $\left(\mathrm{PT} \mathrm{n}^{\circ} 30\right)$. La loi naturelle, comme loi de Dieu connue par la raison dans sa saisie de la nature humaine, est donc le fondement ultime des droits de l'homme qui, en régime chrétien, sont donc à comprendre comme une manifestation de la vocation morale ${ }^{41}$ de l'homme. Une telle fondation des droits de l'homme par la «loi naturelle» en énonce à la fois l'universalité et la pérennité. Leur conformité avec la nature humaine les rend applicables à tout homme, au-delà de toutes les différences. Ils sont l'expression de la parenté profonde qui relie les êtres humains, et en tant que tels le fondement véritable d'une communauté mondiale transcendant tous les particularismes.

En doctrine catholique, l'universalité des droits de l'homme est donc fondée en raison de manière à pouvoir être revendiquée par tous à partir de la nature propre de la personne humaine, de celle de la société et de l'Etat. C'est en tant que ces droits sont reconnus comme l'expression de la loi de Dieu qu'ils sont vraiment ce qu'ils annoncent vouloir être. Néanmoins, il reste un problème avec ce principe d'universalité de la loi naturelle qu'a bien vu le Père S. Pinckaers : c'est celui de la tentation du rationalisme «qui consiste précisément à croire qu'il faille ramener toute la morale à des lois rationnelles applicables et accessibles même aux nonchrétiens pour assurer leur universalisme, et cela jusqu'à écarter les éléments spécifiquement chrétiens qui apparaissent alors comme particuliers. » ${ }^{42}$ Pour éviter ce danger, l'Eglise a articulé à cette universalité rationnelle, l'universalité propre à la loi nouvelle : « soit la puissance de l'Esprit Saint agissant par la foi au Christ et la charité, selon la définition de la Loi nouvelle ${ }^{43}$. Elle a développé, dans le cadre de cet «universalisme spirituel ${ }^{44}$, le concept théologique de la dignité humaine pour dire l'universalité des droits de l'homme.

Dans l'engagement de l'Eglise en faveur des droits de l'homme, on parle à la suite de Jean-Paul II d'une «pastorale des droits humains » 45 fondée sur l'affirmation chrétienne «de la vérité sur l'homme, de l'égale dignité de toute personne, homme ou femme, créée à l'image de Dieu et devenue enfant de Dieu dans le Christ. » En épelant, le 4 juillet dernier, les objectifs d'une telle pastorale,

41 PT n 37 : «L'ordre propre aux communautés humaines est d'essence morale. En effet c'est un ordre qui a pour base la vérité, qui se réalise dans la justice, qui demande à être vivifié par l'amour et qui trouve dans la liberté un équilibre sans cesse rétabli et toujours plus humain. »

42 Servais PINCKAERS o.p., "La loi nouvelle et la permanence des lois morales», in S. PINCKAERS et C.-J. PINTO de OLIVEIRA, Universalité et permanence des lois morales, Fribourg/Paris, Editions Universitaires de Fribourg/Cerf, 1986, p. 449.

43 Servais PINCKAERS o.p., art.cit. p. 443.

44 Idem, p. 448. Il s'agit pour S. Pinckaers de l'universalité de la loi nouvelle.

45 JEAN-PAUL II, « Discours aux participants au Congrès mondial pour la pastorale des droits de l'homme », Samedi 4 juillet 1998, texte original français dans l'Osservatore Romano, 5 juillet 1998, repris dans La Documentation Catholique, 4 octobre 1998, n² 2189, p. 802 et suivantes. 
Jean-Paul II lui donnait comme premier objectif de faire que les droits de l'homme soient partout appliqués. Son deuxième objectif consiste à poser les questions fondamentales relatives à la situation de l'homme aujourd'hui, en prenant position par rapport à l'économie mondiale afin que celle-ci « repose sur les fondations de la dignité et des droits de la personne, surtout le droit au travail et la protection du travailleur. " Le troisième objectif est de promouvoir une véritable culture des droits de l'homme par l'éducation afin d'obtenir dans chacune des cultures de l'humanité le respect intégral de ces droits. Enfin, le quatrième objectif est de s'attacher particulièrement à la dimension spirituelle et transcendante de la personne. Ces quatre objectifs mettent en évidence à quel point le respect des droits de l'homme est lié à l'affirmation et à la reconnaissance de la dignité humaine. C'est bien cette dignité humaine qui se présente comme l'absolu irrécusable et indiscutable que la foi au Christ met sous les yeux pour soutenir le respect des droits de l'homme. On voit dès lors, que le concept de dignité humaine fondant une telle pastorale des droits humains est un concept proprement théologique.

Le terme de dignité humaine connaît une fortune considérable depuis les textes de Vatican II ( Gaudium et spes, Dignitatis humanae) et Pacem in terris. Comme l'a bien vu René Simon, le concept de dignité humaine qu'on retrouve tant au fondement de la Déclaration de l'ONU que des textes magistériels, met en relief trois caractéristiques : "la valeur intrinsèque de la personne, l'appel à l'estime en raison même de cette valeur, une priorité indéductible ${ }^{46}$. Mais est-ce la conscience de la dignité de la personne qui fait sa dignité ? Est-ce la dignité qui conduit nécessairement à en avoir conscience ? La Déclaration de 1948 ne répond pas à la question. Comment alors s'assurer que la dignité dont chacun porte en soi «l'idéal » soit également enracinée dans la réalité de notre vie ? Si le terme « dignité humaine » a l'immense avantage de constituer un "forum commun » de compréhension anthropologique entre croyants et incroyants, il ne faut pas se méprendre sur ses différentes acceptions ${ }^{47}$. Toutes ne sont pas compatibles avec l'anthropologie théologique.

Si la dignité humaine peut être affirmée avec vigueur et se présenter comme une exigence qui demande à s'effectuer, c'est en raison de sa justification par une théologie de la création et de l'incarnation comme on peut le voir dans Pacem in

46 René SIMON, «Le concept de dignité de l'homme en éthique» in A, HOLDEREGGER, R. IMBACH, R. SUAREZ de MIGUEL (eds), De Dignitatis Hominis. Mélanges offerts à C.-J. Pinto de Oliveira, Fribourg/Paris, Editions Universitaires de Fribourg/Cerf, 1987, p. 267-278, en particulier p. 266.

47 Cf : Jean-Michel BREUVART, «Le concept philosophique de dignité humaine », Le Supplément. Revue d'éthique et théologie morale, $\mathrm{n}^{\circ}$ 191, décembre 1994, p. 99-129. 
terris 48 . La conception de l'encyclique met au premier plan le thème de «l'imago Dei $»^{49}$ et du salut en Jésus-Christ ${ }^{50}$ pour dire l'excellence de la dignité de chaque être humain. Pour le chrétien, tout être humain appelle un respect inconditionnel parce qu'il est fils ou fille de Dieu dans l'unique Fils bien aimé. Cette référence au Fils fonde une dignité qui ne découle alors pas de qualités ou d'aptitudes spécifiquement humaines telles que liberté, autonomie, raison, perspectives d'avenir, langage, mémoire ${ }^{51}$. Elle est attachée à la condition de créature de l'homme, qui dans sa nudité, son dénuement, y compris en celui qui a perdu « toute apparence humaine », est pourtant homme dans son rapport constitutif à son Dieu et à ses frères en humanité. En celui qui n'a pas encore accédé au langage ou celui qui l'a perdu, en celui dont la liberté est entravée ou diminuée par une cause psychique, physiologique ou par une cause morale, en celui dont l'humanité semble atrophiée par ses forfaits ou une éducation déficiente et perturbée, le chrétien reconnaîtra malgré tout un frère en humanité qui doit être respecté sans condition. Il y a quelque chose d'extrême dans une telle attestation de la dignité humaine. C'est la portée de la parabole du jugement dernier (Mat 25) et du visage du crucifié. C'est dans la foi et les sacrements que nous percevons l'énigme de la valeur de tout être humain, véritable icône du Christ. Tel est le paradoxe d'une fondation théologique des droits de l'homme d'affirmer que c'est en Dieu, en Christ que se révèle la valeur que l'homme a en lui-même. Nous ne respectons pas l'homme à cause de Dieu ${ }^{52}$ dans le mouvement d'une obéissance externe. Mais parce que la création, l'incarnation et la présence de l'Esprit en toute personne attestent que l'homme vaut d'être respecté en lui-même et pour lui-même et que Dieu lui-même respecte sa créature inconditionnellement au point de se donner à elle en son Fils, gratuitement et inconditionnellement.

48 Pour une présentation de l'encyclique, voir : René COSTE, Michel DAGRAS, Gérard MATHON, Christian MELLON et Raymond MENGUS, Paix sur la terre. Actualité d'une encyclique, Paris, Centurion, 1992.

49 Pacem in terris, $\mathrm{n}^{\circ} 3:$ : Dieu a créé l'homme intelligent et libre à son image et ressemblance, l'établissant d'une autre essence : il faut les chercher là où Dieu les a inscrites, à savoir dans la nature humaine. "

50 Pacem in terris, $\mathrm{n}^{\circ} 10$ : «Les hommes ont été rachetés par le sang du Christ Jésus, faits par la grâce enfants et amis de Dieu et institués héritiers de la gloire éternelle »

51 On voit à l'heure actuelle des philosophes dans le débat sur l'euthanasie ou l'avortement prendre position sur la définition de la dignité humaine en la vidant de son contenu dès lors qu'une personne de manifeste plus de raison, de mémoire, de communication langagière ou de projets d'avenir. Cf : Peter SINGER, Op. cit., 1997.

$52 \mathrm{Cf}$ : Jean-Pierre JOSSUA, «Dieu est-il indispensable ou inutile à la dignité de l'homme ? » in A, HOLDEREGGER, R. IMBACH, R. SUAREZ de MIGUEL (eds), De Dignitatis Hominis. Mélanges offerts à C.-J. Pinto de Oliveira, Fribourg/Paris, Editions Universitaires de Fribourg/Cerf, 1987, p. 369381. 
La parabole du jugement dernier présente les différentes situations où se joue la dignité de l'homme. Comme le dit René Simon, «c'est dans ce qui la nie qu'elle est entrevue ; et elle se développe en prise de conscience des situations et des conditions aliénantes faites au "pauvre", en résistance aux forces qui les produisent et les maintiennent, en lutte pour les supprimer et faire exister une société plus juste et plus fraternelle. $»^{53} \mathrm{~S}$ 'il est bien vrai que la dignité se révèle par contraste dans la faim qui n'a pas de pain, la soif qui n'a pas d'eau pour l'étancher, la nudité qui n'a pas de quoi se vêtir, les entraves de celui qui est privé de liberté, on peut alors comprendre l'intégrité de l'ensemble des droits humains dans la théorie catholique des droits de l'homme.

Avec une grande clarté le théologien américain David Hollenbach a montré, voilà vingt ans, la cohérence et la logique de l'énonciation des droits et devoirs à partir du centre irréductible du respect de la dignité humaine ${ }^{54}$ Ce qui unit les droits fondamentaux, civils, politiques, économiques et sociaux ensemble dans le schéma de Pacem in terris, c'est la norme du respect de la dignité humaine. Mais cette dignité n'est pas abstraite. Elle se donne à voir dans les conditions concrètes de la vie personnelle, sociale, économique et politique. Ce sont finalement les conditions de réalisation de cette dignité qu'on peut appeler «droits de l'homme ». C'est pourquoi, il n'est pas étonnant, même si Pacem in terris est proche par son style de la Déclaration de 1948, que la liste des droits ne soit pas exactement la même, ni exposée dans le même ordre 55 .

Or la conservation et la promotion de la dignité humaine ne dépend pas seulement de l'assouvissement des besoins fondamentaux de l'homme ainsi que de la protection de ses libertés fondamentales. Il faut que les institutions de la société et le pouvoir politique soient ordonnés à la protection de la dignité personnelle. C'est pourquoi, l'encyclique formule ces différents droits comme étant à la fois personnel, social et institutionnel. Ces trois niveaux de droits sont liés au respect effectif de la dignité humaine dans la société. Les droits personnels correspondent aux besoins fondamentaux, libertés et relations qui caractérisent la personne et sans lesquels la dignité humaine ne serait pas préservée. Les droits sociaux (ex : liberté d'expression, éducation, association, soins, réunion...) sont l'expression des formes d'interdépendance qui sont indispensables à la réalisation de la dignité humaine. Ils

53 René SIMON, art. cité, p. 277.

54 David HOLLENBACH, s.j., Claims in Conflict, New York, Ramsey, Paulist Press, 1979, spécialement la reprise d'une étude et d'un diagramme publié par D. CHRISTIANSEN, R. GARET, D. HOLLENBACH and C. POWERS, «Moral Claims, Human Rights and Population Policies", Theological Studies, vol. 35, 1974, p. 102.

55 Cf : l'étude comparative de René COSTE «La première charte ecclésiale des droits de l'homme ", in René COSTE, Michel DAGRAS, Gérard MATHON, Christian MELLON et Raymond MENGUS, Paix sur la terre. Actualité d'une encyclique, Paris, Centurion, 1992, en particulier p.107111. 
représentent les conditions sociales nécessaires à la conservation du bien-être de la personne et spécifient les obligations positives de la société à l'égard de tous. A la différence des droits de l'homme formulés par la tradition séculière occidentale, les droits fondamentaux énumérés par les textes magistériels et tout particulièrement dans Pacem in terris protègent la personne humaine dans les cellules de sa socialité naturelle et dans sa dignité absolue. Parce que l'interdépendance est un aspect essentiel de la vie humaine, la conservation de la dignité humaine est une tâche sociale. Enfin, si l'existence sociale est dessinée et structurée par des institutions telles que l'Etat, le droit, l'économie, le système de santé... la manière dont sont organisées ces institutions revêt une grande importance pour la réalisation concrète des droits personnels et sociaux. L'encyclique a donc énuméré un certain nombre de conditions qui doivent être remplies au sein de ces institutions (les droits instrumentaux) pour que la dignité humaine soit sauvegardée.

Cette distinction des droits fournit une clé de lecture de cette pensée systématique et «intégrale» des droits de l'homme. L'avantage d'une telle distinction est évident pour rendre compte de l'historicité des droits de l'homme et de l'immutabilité du respect de la dignité humaine. Alors que les trois niveaux de droits permettent de reconnaître les différents degrés de contingence historique dans le développement de ces droits, le coeur des droits personnels permet de souligner la pérennité de la défense de la dignité humaine. Mais la vérité de ce qui se joue au niveau éthique dans le respect de la dignité humaine dépend du point de départ théologique.

\section{Conclusion}

Nous voici donc dans une curieuse situation : cherchant à attirer l'attention sur la valeur universelle de tous les êtres humains, nous avons exposé le fondement théologique que lui donne l'enseignement social de l'Eglise. La foi chrétienne nourrit la conviction que nul ne peut jamais dénier à un être humain la valeur constitutive que Dieu a octroyée à chacun et qu'il n'aliène jamais. Elle garantit les droits de l'homme par sa référence à l'amour divin qui nous fonde et nous recrée toujours à nouveau. D'où le paradoxe : la foi chrétienne prétend donner toute leur portée universelle aux droits de l'homme, mais pour y accéder, il faut adhérer à la particularité de la foi.. Est-ce à dire que nous aurions finalement "récupéré » les droits de l'homme au profit du christianisme?

$\mathrm{Au}$ terme de tout ce parcours, une chose est claire : on ne peut pas énoncer la vérité et l'absoluité des droits de l'homme sans affronter la question de leur fondement. En 1948, la Déclaration universelle déclare qu'il est raisonnable de penser que l'homme ne peut exister vraiment que là où une promesse d'existence lui est faite. Or, cette vérité de la précédence d'une promesse qui peut être lue anthropologiquement appartient à la tradition catholique qui ne cesse de lier la 
question de l'homme à l'affirmation de l'amour prévenant de Dieu. Cette promesse de vie pour chacun et tous n'a son sens véritable que si ce n'est pas nous qui l'avons inventée. La Révélation nous livre cette idée-force que l'humanité, dans toute sa diversité et ses infirmités morales, n'en n'est pas moins dotée d'une dignité inaliénable. Pourtant cette vérité que nous proclamons comme faisant partie de notre foi, n'est pas notre exclusivité ni en tant qu'idée, ni en tant qu'expérience.

Si on admet que l'universalité des droits de l'homme peut être reconnue par tous, la foi n'est pas pour autant disqualifiée pour assurer l'exigence du respect de la dignité humaine. Sa contribution n'est pas petite, c'est celle de la vigilance. Une vigilance prophétique qui appelle à temps et à contretemps à voir tout être humain unique et singulier dans et à travers le regard de Dieu car il est tentant, même par le recours à la loi naturelle, de ne fonder le respect des droits de l'homme que sur une humanité «vraie» définie par les qualités positives reconnues à la nature de l'homme. Le respect de la personne est alors suspendu à la reconnaissance de telles qualités. Or trop de nos contemporains n'en ont pas fini avec la conviction que certains auraient perdu toute dignité. Parce que tout homme est Fils ou Fille de Dieu en Jésus-Christ, c'est une dignité absolue qui définit la personne dans son être même et dans ses relations avec autrui. Une telle dignité exige un respect inconditionnel. Le sens de la dignité s'attache à la personne comme telle, y compris quand devenu cadavre inerte, elle est entièrement remise entre les mains d'autrui dans les rites funéraires qui témoignent à l'être disparu un dernier signe de respect.

L' ' universalité spirituelle» de la dignité conférée à tout homme nous place devant un paradoxe : parce qu'elle procède de la foi, elle peut être ignorée par beaucoup. Affirmation "particulière» du respect universellement dû à tout être humain, la foi chrétienne désigne la dignité humaine comme un "universel en contexte» ou selon la formule de Paul Ricoeur, d'un «universel inchoatif » 56 L'avantage d'un tel concept de l'universel, c'est qu'il peut rendre compte de la potentialité de reconnaissance de la dignité humaine dans l'histoire des hommes. Premièrement en soulignant que le respect de la dignité de tout homme ne prend tout son sens qu'en s'inscrivant dans l'histoire des hommes. L'effectuation lui est nécessaire pour l'arracher à l'abstraction d'un principe formel. C'est finalement de s'effectuer que cet a priori, se précise, se fait connaître et reconnaître en sa vérité. Deuxièmement en permettant au mieux de rendre compte de l'articulation entre l'universalité et la pérennité de la dignité humaine et de l'historicité des formulations particulières de son respect. Mais c'est dire qu'une éducation à ce respect, qu'une éducation aux droits de l'homme apparaît comme une exigence première de leur avenir.

56 Cf : Paul RICOEUR, Soi-même comme un autre, Paris, Le Seuil, 1990, p. 336. 
Tékhne, 2010, Vol VIII, $\mathrm{n}^{\circ} 13$

Geneviève Médevielle

\section{Nota Curricular}

Geneviève Médevielle, tem desenvolvido a sua actividade académica no Instituto Católico de Paris (Universidade Católica de Paris) - além de ser convidada noutra Univerdades em França e nos estados Unidos - assumindo as funções de Vice Reitora do Instituto Católico de Paris e Vice-Reitora para a Investigação. Os seus centros de interesse na investigação situam-se, entre outros, no campo dos Direitos do Homem, da ética social e política. Doutorada em ética com uma Tese sobre Ernst Troeltsch que foi galardoada com o Prémio Maurice e Jean de Plange em 1998. Doutorada em ética Teológica tem Agregação em Geografia e é Professora Catedrática da Cadeira Jean-Rodhain.

Tem publicado dezenas de artigos e livros em várias línguas e em diferentes países. Entre as suas obras destacamos L'Absolu au coeur de l'histoire : La notion de compromis chez Ernst Troeltsch, Verf 1998. 